\title{
Controlled decoherence in a quantum Lévy kicked rotator
}

\author{
Henning Schomerus \\ Department of Physics, Lancaster University, Lancaster, LA1 4YB, UK \\ Eric Lutz \\ Department of Physics, University of Augsburg, D-86135 Augsburg, Germany
}

(Dated: March 17, 2008)

\begin{abstract}
We develop a theory describing the dynamics of quantum kicked rotators (modelling cold atoms in a pulsed optical field) which are subjected to combined amplitude and timing noise generated by a renewal process (acting as an engineered reservoir). For waiting-time distributions of variable exponent (Lévy noise), we demonstrate the existence of a regime of nonexponential loss of phase coherence. In this regime, the momentum dynamics is subdiffusive, which also manifests itself in a non-Gaussian limiting distribution and a fractional power-law decay of the inverse participation ratio. The purity initially decays with a stretched exponential which is followed by two regimes of power-law decay with different exponents. The averaged logarithm of the fidelity probes the sprinkling distribution of the renewal process. These analytical results are confirmed by numerical computations on quantum kicked rotators subjected to noise events generated by a Yule-Simon distribution.
\end{abstract}

PACS numbers: $03.65 . \mathrm{Yz}, 05.40 . \mathrm{Fb}, 72.15 . \mathrm{Rn}$

\section{INTRODUCTION}

The coupling of an open quantum system to an external reservoir induces a dynamical loss of phase coherence that results in an irreversible destruction of interference effects between states of the system [1, 2]. This environment-induced decoherence efficiently suppresses macroscopic superpositions of states and therefore plays a central role in our understanding of the transition from quantum to classical physics. An important and often annoying feature of decoherence for quantum-mechanical applications is that it typically occurs very rapidly with an exponential decoherence factor, $\mathcal{D}(t)=\exp \left(-t / t_{c}\right)$, where $t_{c}$ is the short coherence time $[1,2]$. In some cases, however, the coherence time can be controlled by properly engineering the reservoir and/or the interaction between the system and the reservoir [3]. The feasibility of such schemes has been experimentally demonstrated in a variety of systems that include cavity QED [4], ion traps [5] and cold atom experiments [6-8]. Besides their obvious technological implications, these experiments provide essential tests of the general concept of environmentinduced decoherence.

The atom-optical experiments [6-8] are designed to realize the quantum-kicked rotator [9-11], a paradigm of complex chaotic dynamics which can be simply seen as a particle moving on a ring and periodically kicked in time. For sufficiently strong kicking strength, the classical motion of a kicked rotator is chaotic and the evolution in momentum space is diffusive. In the quantum regime, however, momentum diffusion is asymptotically suppressed due to dynamical localization $[12,13]$. In the recent atom-optical reservoir-engineering experiments, the loss of coherence has been induced by amplitude noise [7] and timing noise [8]. In the first case, the amplitude of the periodic kicks is randomly modu- lated in a controlled manner, whereas in the second case the period between successive kicks is randomly varied. In both situations, dynamical localization is extenuated leading to a non-vanishing quantum momentum diffusion with a renormalized diffusion coefficient, in agreement with theoretical predictions $[7,14,15]$. These experiments enjoy a high degree of control and tunability, which makes the atom-optical setup ideally suited to investigate the decoherence-induced quantum-to-classical crossover of complex quantum systems [16].

One point is especially noteworthy: reservoir engineering has so far been used to tune the coherence time, but not the exponential time dependence of the decoherence factor $\mathcal{D}(t)$ itself. Our aim in this paper is to describe a reservoir coupling scheme for the cold atom experiments which allows to modify the time dependence of $\mathcal{D}(t)$ and slow down the loss of phase coherence in a controlled way. To this end, we superpose the regular periodic pulses with noise events occurring with randomly modulated time intervals $\tau$ (i.e., we combine amplitude with timing noise). We generate these random time intervals by a renewal process with a waiting-time distribution $w(\tau)$. Of special interest is the case where this distribution asymptotically behaves as a power law, $w(\tau) \propto \tau^{-1-\alpha}$. By tuning the exponent $\alpha$ of this so-called Lévy noise [17], we are able to change the mean waiting time between successive noise events, given by $\bar{\tau}=\int_{0}^{\infty} d \tau \tau w(\tau)$, and therefore can smoothly interpolate between a fully coupled situation where most kicks are perturbed by the noise (large $\alpha)$ to an almost isolated situation where most kicks are not perturbed by the noise $(\operatorname{small} \alpha)$. Divergent moments are a hallmark of Lévy statistics and the mean waiting time $\bar{\tau}$ becomes infinite when $\alpha \leq 1$. In this case the noise is nonstationary and nonergodic $[18,19]$. We show that this type of noise modifies the decoherence of the atoms in a striking manner: The exponential time dependence 
of the decoherence factor $\mathcal{D}(t)$ is replaced by a MittagLeffler function [20], which starts out as a stretched exponential and asymptotically decays as a power-law. For this functional form, the coherence time is ill-defined.

In Ref. [21] we briefly discussed one manifestation of the modified decoherence pattern, namely a subdiffusive momentum spreading of the atoms. In this work, we present a theory that links the subdiffusive behavior to the nonstationarity of the noise. This requires us to extend the previous theories of Refs. [14, 15] beyond the perturbative short-time regime and to account for the additional timing noise from the renewal process. We also discuss the limiting distribution function and the inverse participation ratio, which provide additional information about the dynamics in momentum space. As alternative measures of coherence, we then consider the purity and the fidelity [22-24] of atoms subjected to different realizations of the noise, which can be probed in an echo experiment, such as carried out, e.g., for microcavities in Ref. [25] (for atom-optical proposals of such experiments see Refs. [26-28]). For nonexponential decoherence, we find that the purity initially decays with a stretched exponential, which is followed by two regimes of power-law decay with different exponents. The decay of the averaged logarithm of the fidelity is related to the sprinkling distribution of the renewal process. Throughout the paper our findings are illustrated by comparison to the results from a numerical implementation of the kicked rotator.

The structure of this paper is as follows. Section II describes the quantum kicked rotator and the environmental coupling scheme of combined amplitude and timing noise generated by a renewal process. In Sec. III we derive the nonperturbative expression for the decoherence factor and evaluate it for Lévy noise. In the case of nonstationary noise with $\alpha \leq 1$, the decoherence function is nonexponential. Section IV discusses how the modified decoherence affects the momentum spreading, while Sec. $\mathrm{V}$ contains the discussion of the purity and fidelity. The results of this paper are summarized in Sec. VI.

\section{THE LÉVY KICKED ROTATOR}

\section{A. Model}

The motion of atoms exposed to pulsed standing-wave potentials can be mapped onto a quantum kicked rotator [9-11], i.e., a point particle moving on a ring and subjected to periodical kicks. In suitable units, the Hamiltonian of the kicked rotator takes the form

$$
H=\frac{p^{2}}{2}+\sum_{n=-\infty}^{\infty} K_{n} \cos \theta \delta\left(t-n+0^{+}\right),
$$

where the kicking potential depends on the $2 \pi$-periodic rotation angle $\theta$ and the kicking amplitude $K_{n}$ can in general be kick dependent.
From kick to kick, the stroboscopic classical dynamics of the kicked rotator is generated by the map

$$
\begin{aligned}
& p(t+1)=p(t)+K_{t} \sin \theta, \\
& \theta(t+1)=\theta(t)+p(t+1) .
\end{aligned}
$$

Starting from a given initial state $\psi(0)$, the corresponding quantum dynamics $\psi(t+1)=F\left(K_{t}\right) \psi(t)$ is generated by the Floquet operator

$$
F\left(K_{t}\right)=\exp \left(-i \hbar^{-1} \hat{p}^{2} / 2\right) \exp \left(-i \hbar^{-1} K_{t} \cos \hat{\theta}\right) .
$$

In both cases, the dynamics consists of a kick in which the momentum changes by $K_{t} \sin \theta$, followed by a free rotation in which the rotation angle $\theta$ increases by $p$.

In the absence of noise, the dynamics of the kicked rotator is entirely controlled by the constant parameter $K_{n}=K$. For $K \gtrsim 5$, the classical dynamics is chaotic and the growth of the momentum is on average diffusive with a variance $\operatorname{var} p(t) \simeq D_{\mathrm{cl}} t$. The classical diffusion constant is given by $D_{\mathrm{cl}} \simeq K^{2} / 2$ [29]. By contrast, in the quantum regime, the kicked rotator exhibits dynamical localization, an interference phenomenon akin to Anderson localization in disordered solids, which manifests itself in an exponentially decaying envelope of the quasienergy eigenstates $F(k)$ of the Floquet operator [12]. As a result, momentum diffusion is suppressed and the variance

$$
\operatorname{var} p_{0}(t) \simeq D^{*} t^{*}\left[1-\exp \left(-t / t^{*}\right)\right]
$$

saturates after the quantum break time $t^{*} \simeq D^{*} / \hbar^{2}[13]$. The constant $D^{*}$ is of order of the classical diffusion constant $D_{\mathrm{cl}}$, but is subject to quantum corrections [30].

The transition between the quantum and the classical behavior of the rotator can be induced by subjecting the kicked particle to additional random kicks, simulating in such way the coupling to an external environment, where the characteristics of the imposed noise is controlled externally. In the case of amplitude noise [7], the kicking amplitude is explicitly $n$-dependent, $K_{n}=K+k_{n}$, where the perturbations $k_{n}$ are random numbers. In the presence of conventional timing noise [8] the period between the kicks (here set to unity) is slightly modulated.

In this paper we consider an unconventional combination of amplitude and timing noise: kicks always appear at periodic instances and the amplitude of some of the kicks are perturbed. However, the (integer-valued) time intervals between the noise events are generated by a renewal process with waiting-time distribution $\omega(\tau)$ (see Fig. 1 for a schematic illustration). The timing of the $\mathcal{N}$ 'th noise event $(\mathcal{N}=1,2,3, \ldots)$ is hence given by

$$
t_{\mathcal{N}}=\sum_{n=1}^{\mathcal{N}} \tau_{n}
$$

where each waiting time $\tau_{n}$ is drawn independently from the same probability distribution $\omega(\tau)$. The strength of the individual noise events will be characterized by the 
(a)

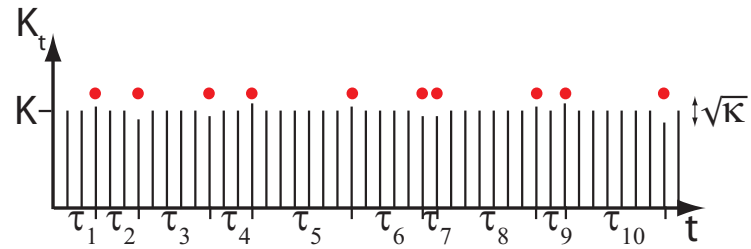

(b)

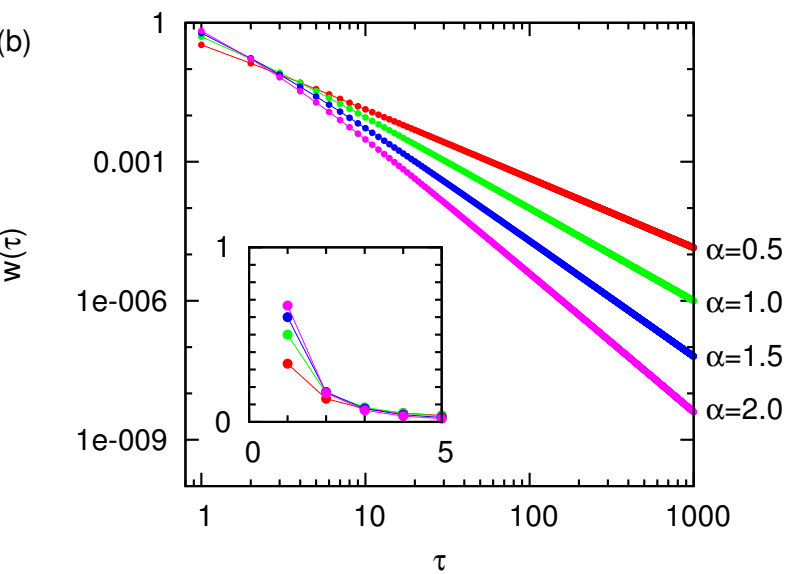

FIG. 1: (color online). (a) Schematic illustration of the kicking sequence in the Lévy kicked rotator. The period of the kicks is set to unity. The timing of the noise is generated in a renewal process, which selects the integer-valued waiting times $\tau_{n}$ from a distribution $\omega(\tau)$. (b) Waiting-time distributions of the Yule-Simon form (24) for different values of the parameter $\alpha$. The distributions display power-law tails, specified in Eq. (25), and hence generate Lévy noise of variable exponent. The lines are a guide to the eye.

variance $\overline{k_{t_{\mathcal{N}}}^{2}}=\kappa$ of the perturbed kicks, whereas on average $\overline{k_{t_{\mathcal{N}}}}=0$.

The usual white amplitude noise is obtained for a waiting-time distribution $\omega(\tau)=\delta_{\tau, 1}$, so that every kick is perturbed. A more intriguing case is a waiting-time distribution which asymptotically behaves as a power law (Lévy noise),

$$
w(\tau) \sim c \tau^{-1-\alpha}
$$

where $c$ is a constant. For $\alpha<1$ the mean waiting time $\bar{\tau}$ diverges. Unlike the familiar white amplitude noise, Lévy noise [31] generates a nonstationary process with unconventional statistical features that are reviewed in the following subsection.

\section{B. Statistical properties of Lévy noise}

The properties of noise generated by a renewal process can be conveniently quantified by analyzing the number of noise events $\mathcal{N}\left(t^{\prime}, t^{\prime \prime}\right)$ within an interval $\left[t^{\prime \prime}, t^{\prime}\right)$. The number $\mathcal{N}(t, 0)$ is obtained by inverting the random time $t_{\mathcal{N}}$, and hence is known as the inverse random time of the process [32]. On average, the rate of change $f(t)=\partial_{t} \overline{\mathcal{N}(t, 0)}$ of the inverse random time gives the probability that there is a noise event at time $t$. This rate is called the sprinkling distribution and is directly related to the waiting-time distribution $\omega(\tau)$ via a Laplace transformation,

$$
\tilde{f}(u)=\int_{0}^{\infty} d t e^{-u t} f(t)=\frac{\tilde{w}(u)}{1-\tilde{w}(u)} .
$$

We denote throughout the article Laplace-transformed functions by a tilde.

For small values of $u$, the power-law waiting-time distribution (7) delivers

$$
\begin{aligned}
\tilde{w}(u) & \sim 1-u \bar{\tau} & & (\alpha>1), \\
\tilde{w}(u) & \sim 1-u^{\alpha} \frac{c \pi}{\Gamma(\alpha+1) \sin \pi \alpha} & & (\alpha<1) .
\end{aligned}
$$

For $\alpha>1$, it then follows from Eq. (8) that the sprinkling distribution takes a constant value at large times,

$$
f(t) \sim \frac{1}{\bar{\tau}} \quad(\alpha>1)
$$

As a result, the noise asymptotically becomes stationary in this case. However, for power-law waiting-time distributions with $\alpha<1$, the sprinkling distribution asymptotically decays over time according to

$$
f(t) \sim t^{\alpha-1} \alpha \sin (\pi \alpha) /(\pi c) \quad(\alpha<1) .
$$

Due to the explicit time dependence of $f(t)$, the noise here remains nonstationary. We note that nonstationary also implies nonergodicity, since time averages cannot be replaced by ensemble averages [19]. A direct consequence of Eq. (12) is that the mean inverse random time increases sublinearly,

$$
\overline{\mathcal{N}(t, 0)} \sim t^{\alpha} \sin (\pi \alpha) /(\pi c) \quad(\alpha<1) .
$$

More detailed information about the statistical properties of the renewal process can be obtained by analyzing the complete distribution function $\mathcal{P}\left(\mathcal{N} ; t^{\prime}, t^{\prime \prime}\right)$ of the number of noise events. The latter is most conveniently characterized by the moment-generating function

$$
\mathcal{M}\left(z ; t^{\prime}, t^{\prime \prime}\right)=\overline{\exp \left[z \mathcal{N}\left(t^{\prime}, t^{\prime \prime}\right)\right]}=\sum_{n=0}^{\infty} e^{z \mathcal{N}} \mathcal{P}\left(\mathcal{N} ; t^{\prime}, t^{\prime \prime}\right)
$$

For general time arguments $t^{\prime}$ and $t^{\prime \prime}$, this function can be expressed as

$$
\begin{aligned}
\mathcal{M}\left(z ; t^{\prime}, t^{\prime \prime}\right)= & \mathcal{M}\left(z ; t^{\prime}, 0\right) \\
& -\left(e^{z}-1\right) \int_{0}^{t^{\prime \prime}} d s f(s) \mathcal{M}\left(z ; t^{\prime}-s, 0\right),
\end{aligned}
$$

so that it suffices to study the moment-generating function of the inverse random time $\mathcal{N}(t, 0)$.

We first relate the distribution function $\mathcal{P}(\mathcal{N} ; t, 0)$ of the inverse random time to the probability distribution 
$P\left(t_{\mathcal{N}} ; \mathcal{N}\right)$ of the $\mathcal{N}$ th random time, which succeeds via the intuitive expression

$$
\mathcal{P}(\mathcal{N} ; t, 0)=\int_{0}^{t} d t^{\prime}\left[P\left(t^{\prime} ; \mathcal{N}\right)-P\left(t^{\prime} ; \mathcal{N}+1\right)\right] .
$$

Since Eq. (6) involves a sum of $\mathcal{N}$ independent waiting times, the Laplace transform of $P\left(t_{\mathcal{N}} ; \mathcal{N}\right)$ factorizes,

$$
\tilde{P}(u ; \mathcal{N})=[\tilde{w}(u)]^{\mathcal{N}},
$$

and Eq. (16) thus yields

$\widetilde{\mathcal{P}}(\mathcal{N} ; u, 0)=\int_{0}^{\infty} d t e^{-u t} \mathcal{P}(z ; t, 0)=\frac{1}{u}[1-\tilde{w}(u)][\tilde{w}(u)]^{\mathcal{N}}$.

(As indicated, the Laplace transformation is taken with respect to the later time argument). By using the definition (14) of the moment-generating function $\mathcal{M}(z ; t, 0)$, we find that its Laplace-transform can be expressed as a function of the sprinkling distribution,

$$
\widetilde{\mathcal{M}}(z ; u, 0)=\frac{1}{u} \frac{1}{1-\tilde{f}(u)\left(e^{z}-1\right)} .
$$

One should note the explicit periodicity, $\widetilde{\mathcal{M}}(z ; u, 0)=$ $\widetilde{\mathcal{M}}(z+2 \pi i ; u, 0)$, which is a consequence of the discreteness of the inverse random time.

When next going back to the time domain, we again have to distinguish between asymptotically stationary and nonstationary noise. In the former case $(\alpha>1)$, the moment-generating function is asymptotically exponential,

$$
\mathcal{M}(z ; t, 0) \sim \exp \left[\left(e^{z}-1\right) t / \bar{\tau}\right] \quad(\alpha>1) .
$$

Equation (20) characterizes the counting statistics of a Poisson process with mean rate $\bar{\tau}^{-1}$. In the nonstationary case $\alpha<1$, inserting the asymptotic behavior (10) produces a moment-generating function of the form

$\mathcal{M}(z ; t, 0) \sim E_{\alpha}\left[t^{\alpha}\left(e^{z}-1\right) \Gamma(1+\alpha)(\sin \pi \alpha) /(\pi c)\right] \quad(\alpha<1)$,

where $E_{\alpha}$ is the Mittag-Leffler function [20], defined by

$$
E_{\alpha}(z)=\sum_{n=0}^{\infty} \frac{z^{n}}{\Gamma(\alpha n+1)}
$$

Expression (21) becomes exact for all times when one passes to continuous inverse times $\left(e^{z}-1 \rightarrow z\right)$ and considers the particular case of a scale-free Lévy process [32]. The moment-generating function then starts out as a stretched exponential, $\mathcal{M}(z ; t, 0) \simeq \exp \left\{t^{\alpha} /\left[\Gamma(-\alpha) c t_{c}\right]\right\}$, while for large times it crosses over to the power law $\mathcal{M}(z ; t, 0) \simeq\left(c t_{c} / \alpha\right) t^{-\alpha}$. For more general waiting-time distributions, a reasonable approximation is to adapt the time argument by using the mean inverse random time, such that

$$
\mathcal{M}(z ; t, 0) \approx E_{\alpha}\left[\left(e^{z}-1\right) \Gamma(1+\alpha) \overline{\mathcal{N}(t, 0)}\right] \quad(\alpha<1) .
$$

This results in the same power-law asymptotics for large times, and accounts for transient behavior in the initial decay.

\section{Numerical implementation}

Throughout this paper we compare analytical results to results obtained from a numerical implementation of the Lévy kicked rotator. In the present section, we describe the parameters used for these computations.

Dynamical localization is expected to be the strongest when $\hbar /(2 \pi)$ is approximating a quadratic irrational $\omega=$ $\frac{1}{2}\left(\sqrt{a^{2}+4}-a\right)$. In order to render the Hilbert space finite we choose $a=24$, set $\hbar=2 \pi M / N$, and use the third convergent of the continued-fraction representation of the quadratic irrational, $\frac{1}{24+} \frac{1}{24+} \frac{1}{24}$. This gives $M=577$, $N=13872$.

The integer $N$ is the Hilbert space dimension, which now is large but finite, corresponding to a quantized momentum $p=\hbar l, l=0,1,2, \ldots N-1$. Similarly, in position space the momentum quantization carries over to discretized positions $\theta=2 \pi l / N$. The integer $M$ determines the quantum-mechanical periodicity in momentum space ( $p$ and $p+2 \pi M$ are equivalent). Each classical period of $2 \pi$ covers $N / M \approx 24$ discretized momentum values.

In all our computations, the particle is initially prepared in the zero-momentum state $\psi(0)=|p=0\rangle$. The subsequent propagation is obtained by successive application of the Floquet operator (4), which can be broken down into the application of diagonal matrix multiplications in momentum and position representation, intervened by Fast Fourier Transformations for the passage between both representations.

In each propagation step, the kicking strength is determined according to the following procedure. The regular kicking strength is set to $K=7.5$. The noisy perturbations $k_{n}$ are taken from a uniform box distribution over an interval $(-W, W)$, so that $\kappa=W^{2} / 3$. This noise is only applied at times selected by a renewal process, which we generate by the so-called Yule-Simon distributions [33]

$$
\omega(\tau)=\frac{\alpha \Gamma(\tau) \Gamma(\alpha+1)}{\Gamma(\tau+\alpha+1)} .
$$

These distributions are paradigms of integer-valued probability distributions with a power-law tail,

$$
\omega(\tau) \sim \alpha \Gamma(\alpha+1) \tau^{-1-\alpha} .
$$

The mean waiting time is given by

$$
\bar{\tau}=\frac{\alpha}{\alpha-1} \quad(\alpha>1)
$$

and diverges for $\alpha<1$. In the latter case, the asymptotic time dependence of the sprinkling distribution is

$$
f(t) \sim \frac{\sin (\pi \alpha)}{\pi \Gamma(\alpha+1)} t^{\alpha-1} .
$$

These properties directly follow from the Laplace transform of the waiting-time distribution, which reads

$$
\tilde{w}(u)=\frac{\alpha}{\alpha+1} e_{2}^{-u} F_{1}\left(1,1, \alpha+2 ; e^{-u}\right),
$$




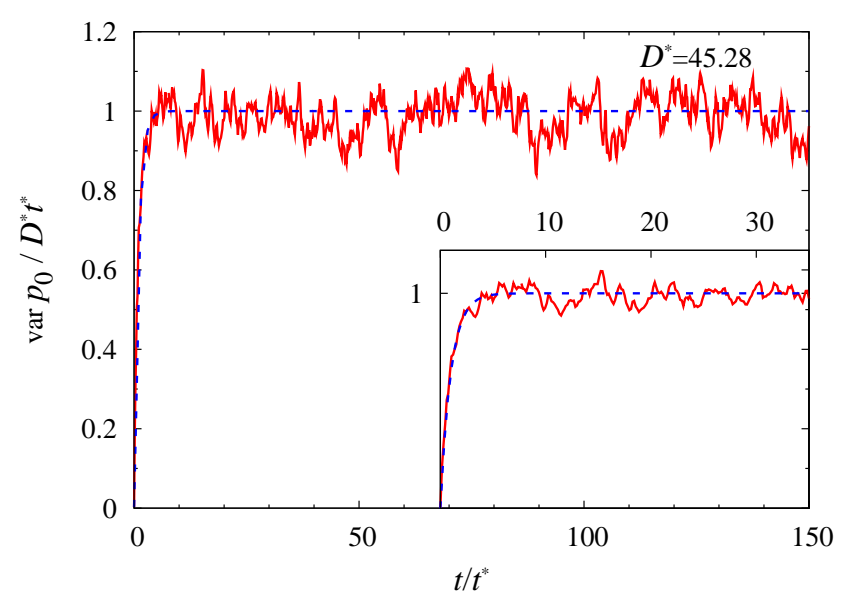

FIG. 2: (color online). Time dependence of the variance of momentum var $p_{0}(t)$ for a noiseless kicked rotator with regular kicking strength $K=7.5$. The dashed curve is the theoretical prediction (5) of dynamical-localization theory [13]. A singleparameter fit delivers the value $D^{*}=\hbar^{2} t^{*}=45.28$, which is used throughout the rest of the present work. The inset zooms in onto the region where dynamical localization is established.

where ${ }_{2} F_{1}$ is the hypergeometric function.

The variance of the momentum for the kicked rotator in the absence of noise is shown in Fig. 2. A single-parameter fit to the prediction (5) of dynamicallocalization theory delivers the value $D^{*}=\hbar^{2} t^{*}=45.28$, which is our only fitting parameter and will not be adjusted any further throughout the rest of this work.

\section{DECOHERENCE IN QUASIENERGY SPACE}

The effect of noise on the quantum kicked rotator has been first investigated by Ott, Antonsen and Hanson, who developed the following intuitive picture [14]. In the absence of noise the quantum system is fully coherent: the quasienergy eigenstates of the Floquet operator $F(K)$ are exponentially localized in momentum space, chaotic diffusion is strongly suppressed and the momentum diffusion constant asymptotically vanishes. The effect of the external noise is to couple the quasienergy states and to induce transitions between them. As a result, dynamical localization is extenuated and quantum diffusion takes place.

A systematic approach for calculating the non-zero quantum diffusion constant in the presence of stationary noise has been developed by Cohen [15]. It is based on an analysis of the decay of a decoherence factor which is related to the survival probability of the quasienergy states of the Floquet operator. In Ref. [15] this decoherence factor was calculated perturbatively for short times (the emphasis was on stationary noise with arbitrary correlations, including the intricate correlations of zero-temperature noise). In the following, we use a simple random-phase approximation to calculate the decoherence factor beyond the perturbative short-time regime. This approximation is valid for random uncorrelated noise events and remains immediately applicable to noise generated by a renewal process, including the case of nonergodic, nonstationary Lévy noise.

\section{A. Quasienergy transitions and decoherence factor}

The quasienergy eigenstates $|r\rangle$ of the noiseless system are defined by the eigenvalue equation

$$
F(K)|r\rangle=\exp \left(-i \varepsilon_{r}\right)|r\rangle
$$

where $\varepsilon_{r}$ is the quasienergy. A noise-free propagation step does not alter the quasienergy state, while a noisy kick introduces transitions with amplitude

$$
\left\langle s\left|F\left(K_{n}\right)\right| s^{\prime}\right\rangle=\exp \left(-i \varepsilon_{s}\right)\left\langle s\left|\exp \left(-i \hbar^{-1} k_{n} \cos \theta\right)\right| s^{\prime}\right\rangle,
$$

where $s^{\prime}$ and $s$ are the quasienergy index before and after the propagation step, respectively.

Over a larger time interval $\left[t^{\prime \prime}, t^{\prime}\right)$, the survival amplitude in a quasienergy state $|r\rangle$ is defined as

$$
A_{r}\left(t^{\prime}, t^{\prime \prime}\right)=\left\langle r\left|\mathcal{T} \prod_{n=t^{\prime \prime}}^{t^{\prime}-1} F\left(K_{n}\right)\right| r\right\rangle \exp \left[i\left(t^{\prime}-t^{\prime \prime}\right) \varepsilon_{r}\right]
$$

where $\mathcal{T}$ is the time-ordering operator. In this expression, we have explicitly provided for two time arguments, as is required when working with a renewal process which may produce nonstationary noise. The exponential factor compensates for the dynamical accumulation of quasienergy phases in the noiseless system, for which $A_{r}\left(t^{\prime}, t^{\prime \prime}\right)=1$.

The averaged survival amplitude is denoted by

$$
\mathcal{D}^{(1)}\left(t^{\prime}, t^{\prime \prime}\right)=\overline{A_{r}\left(t^{\prime}, t^{\prime \prime}\right)},
$$

where the average is over the index $r$ as well as over the timing and amplitude of the noise.

The decoherence factor $\mathcal{D}\left(t^{\prime}, t^{\prime \prime}\right)$ that later appears in the momentum spreading and in the decay of the purity is given by

$$
\mathcal{D}\left(t^{\prime}, t^{\prime \prime}\right)=\overline{A_{r}\left(t^{\prime}, t^{\prime \prime}\right) A_{s}^{*}\left(t^{\prime}, t^{\prime \prime}\right)},
$$

where the average now extends over both quasienergy indices $r \neq s$.

\section{B. Average over amplitude noise}

In order to analyze the decoherence factor, we first assume that the timing of noise events is fixed and average over the random detunings $k_{n}$ of the noise events, as well as over the quasienergy indices. These averages become straightforward within a random-phase approximation, 
which uses that the random detunings are uncorrelated and moreover exploits features of the complex quantum dynamics encoded in the quasienergy transition amplitudes (30). For this we have to distinguish amplitudes which preserve the quasienergy index (diagonal matrix elements, which are of modulus one if there is no noise and less than one in the presence of noise) from amplitudes describing transitions between quasienergy states (off-diagonal matrix elements, which are only finite in the presence of noise).

For noise strength $\kappa \ll \hbar^{2}$ the averaged modulus of the diagonal matrix elements in Eq. (30) can be obtained in a simple expansion,

$$
\overline{\exp \left(-i \hbar^{-1} k_{l} \cos \theta\right)} \approx 1-\left(\kappa / 2 \hbar^{2}\right) \overline{\cos ^{2} \theta}=1-\kappa / 4 \hbar^{2},
$$

which delivers the survival time

$$
t_{c} \simeq 2 \hbar^{2} / \kappa \text {. }
$$

We will shortly see that $t_{c}$ coincides with the decoherence time for conventional amplitude noise, which acts stationarily in every kick. The condition set for the noise strength implies $t_{c} \gg 1$. This assumption is not very restrictive, since it only prevents total decoherence by a single event, but does not impose any restriction on $t_{c}$ with respect to the localization time $t^{*} \simeq K^{2} / 2 \hbar^{2}$, which is also much larger than unity. We hence reserve the notion of weak noise for the more restrictive condition $\kappa \ll \hbar^{4} / K^{2}$, which implies $t_{c} \gg t^{*}$.

Each noise event mixes the initial quasienergy state into about $\xi=t^{*} / 2 \gg 1$ states whose localization centers are in reach of the localization length [30]. Each individual off-diagonal transition amplitude hence is small, and for weak noise $\left(t_{c} \gg t^{*}\right)$ the averaged off-diagonal matrix elements indeed can be trivially neglected. The averaged Floquet operator $F\left(K_{n}\right)$ is then simply approximated by

$$
\left\langle s\left|\overline{F\left(K_{n}\right)}\right| s^{\prime}\right\rangle=\delta_{s, s^{\prime}} \operatorname{diag}\left[e^{-i \varepsilon_{s}-1 /\left(2 t_{c}\right)}\right],
$$

which is diagonal in quasienergy space.

When $t_{c}$ and $t^{*}$ are of comparable order, individual contributions which include quasienergy transitions are still small, but in the composed survival amplitude (31) one cannot disregard the proliferation of such terms when the number of transitions is increased. It then becomes necessary to exploit the consequences of the complex dynamics of the kicked rotator, which induces random phases into the off-diagonal transition matrix elements. The random phase has several separate origins:

(1) For fixed $s$ and $s^{\prime}$, the transition amplitude (30) sensitively depends on the random detuning $k_{n}$ of the kick.

(2) In momentum space, the quasienergy states have an exponentially decaying envelope, but a quasi-random internal structure. Consequently, even after the average over the detuning, the phase of the transition amplitude depends strongly on the quasienergy indices $s$ and $s^{\prime}$.

In the ensuing random-phase approximation, the phase of the off-diagonal transition amplitudes is assumed to be uniformly distributed in $[0,2 \pi)$, while the squared modulus of these matrix elements is of order $2 /\left(t_{c} t^{*}\right)$ (corresponding to the bandwidth $\left.\xi=t^{*} / 2\right)$. The squared modulus of the diagonal elements is given by $\exp \left(-1 / t_{c}\right) \approx$ $1-1 / t_{c}$.

In this approximation, the averaged Floquet operator $F\left(K_{n}\right)$ simply retains its diagonal form (36). This is all we need for the computation of the averaged survival amplitude $\mathcal{D}^{(1)}\left(t^{\prime}, t^{\prime \prime}\right)$ and leads to the intermediate expression

$$
\mathcal{D}^{(1)}\left(t^{\prime}, t^{\prime \prime}\right)=\overline{\exp \left[-\mathcal{N}\left(t^{\prime}, t^{\prime \prime}\right) /\left(2 t_{c}\right)\right]},
$$

where $\mathcal{N}\left(t^{\prime}, t^{\prime \prime}\right)$ is the inverse random time of the renewal process as introduced in Sec. II.

The random phase approximation also establishes a direct connection between $\mathcal{D}^{(1)}\left(t^{\prime}, t^{\prime \prime}\right)$ and the decoherence factor $\mathcal{D}\left(t^{\prime}, t^{\prime \prime}\right)$. Since in Eq. (33) the two quasienergy indices $r$ and $s$ are not identical, there are no contributions which link the quasienergy states in exactly the same sequence. The decoherence factor therefore immediately factorizes into the averaged survival amplitudes of the two quasienergy states, yielding

$$
\mathcal{D}\left(t^{\prime}, t^{\prime \prime}\right)=\overline{\left[\mathcal{D}^{(1)}\left(t^{\prime}, t^{\prime \prime}\right)\right]^{2}}=\overline{\exp \left[-\mathcal{N}\left(t^{\prime}, t^{\prime \prime}\right) / t_{c}\right]} .
$$

The remaining average over the timing of the noise events does not factorize, but because of the simple exponential form of Eq. (37), merely amounts to a factor of two in the exponent. We now analyze this average for different types of renewal processes.

\section{Average over timing noise}

In the conventional case of stationary noise with $\omega(\tau)=\delta_{\tau, 1}$, where timing noise is absent, the inverse random time, $\mathcal{N}\left(t^{\prime}, t^{\prime \prime}\right)=t^{\prime \prime}-t^{\prime}$, does not fluctuate. According to Eq. (38), the decoherence factor

$$
\mathcal{D}\left(t^{\prime}, t^{\prime \prime}\right)=\exp \left[-\left(t^{\prime}-t^{\prime \prime}\right) / t_{c}\right] \quad \text { (stationary noise) }
$$

then decays exponentially over time, and $t_{c}$ is identical to the coherence time.

For noise generated by a renewal process with powerlaw waiting-time distributions, the subsequent analysis depends on whether the process is characterized by a mean waiting time $\bar{\tau}$, or shows the nonstationary features of Lévy noise. This bifurcation can be made explicit by realizing that Eq. (37) is identical to the momentgenerating function $\mathcal{M}\left(z ; t^{\prime}, t^{\prime \prime}\right)$ of the number of noise events, introduced in Eq. (14), which has to be evaluated at the noise-strength dependent value $z=-1 / t_{c}$. We therefore can immediately exploit the results of Sec. II B. (Since we assume $t_{c} \gg 1$ we can set $e^{z}-1 \approx-1 / t_{c}$.)

It follows from Eq. (20) that, when the noise is asymptotically stationary $(\alpha>1)$, the decoherence factor retains its exponential form,

$$
\mathcal{D}\left(t^{\prime}, t^{\prime \prime}\right) \sim \exp \left[-\left(t^{\prime}-t^{\prime \prime}\right) / \bar{\tau} t_{c}\right] \quad(\alpha>1) .
$$



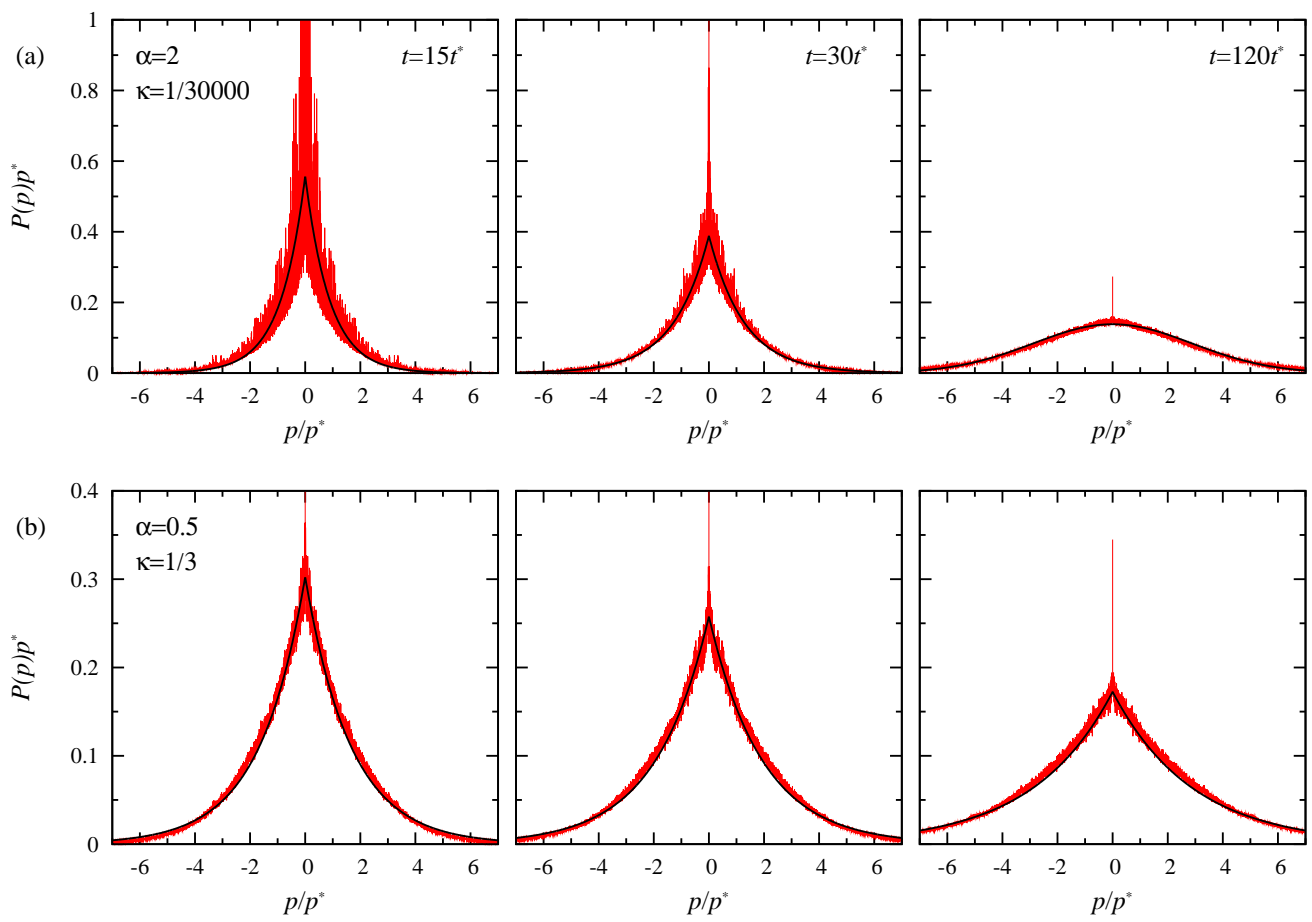

FIG. 3: (color online). Averaged distribution of momenta $P(p ; t)$ at selected times $t$ for kicked rotators which are subjected to Lévy noise generated by a Yule-Simon distribution. The average is over $10^{3}$ realizations. The smooth curves are fits to double-sided exponential and Gaussian distributions. The unit of momentum is the localization length $p^{*}=D^{*} / \hbar$. (a) In the upper panels $\alpha=2.0, \kappa=1 / 30000$, corresponding to a decoherence time $t_{c}=12.3 t^{*}$ (where $t^{*}$ is the localization time). At $t=15 t^{*} \approx 1.2 t_{c}$ the distribution function fluctuates around a double-sided exponential function. At $t=30 t^{*} \approx 2.4 t_{c}$ the distribution function is still exponential, but the fluctuations are suppressed. At $t=120 t^{*} \approx 9.8 t_{c}$ the distribution function is well described by a Gaussian. (b) In the lower panels $\alpha=0.5, \kappa=1 / 3$, corresponding to stronger but nonstationary, increasingly rare noise events. In this case, a decoherence time cannot be defined and the distributions function remains exponential for all times $t>t^{*}$. Note that the peak at $p=0$ arises from coherent backscattering.

The effective coherence time $\bar{\tau} t_{c}$ is directly proportional to the mean waiting time of the noisy kicks and therefore increases with decreasing exponent $\alpha$. In particular, the effective coherence time becomes infinitely large when $\alpha$ drops below unity. According to Eq. (23), the functional form of the decoherence factor then changes to

$$
\mathcal{D}(t, 0) \approx E_{\alpha}\left[-\Gamma(1+\alpha) \overline{\mathcal{N}(t, 0)} / t_{c}\right] \quad(\alpha<1) .
$$

After a (typically short) transient, $\overline{\mathcal{N}(t, 0)} \sim$ $t^{\alpha} \sin (\pi \alpha) /(\pi c)$. Hence, we have the remarkable result that Lévy noise induces a nonexponential loss of coherence which typically starts out as a stretched exponential,

$$
\mathcal{D}(t, 0) \simeq \exp \left[t^{\alpha} /\left(\Gamma(-\alpha) c t_{c}\right)\right], \quad(\text { initial decay })
$$

and for large times crosses over to a power law,

$$
\left.\mathcal{D}(t, 0) \simeq\left(c t_{c} / \alpha\right) t^{-\alpha} \quad \text { (asymptotic decay }\right)
$$

The functional dependence of the decoherence factor can thus be changed in a controlled manner by tuning the value of the exponent $\alpha$. We now explore how this affects the observable properties of the kicked rotator.

\section{MOMENTUM SPREADING}

In the atom-optical experiments, the momentum can be observed by a time-of-flight measurement, where the kicking sequence is terminated at a specified time $t$ and the atom cloud is allowed to expand ballistically to a much larger size. A snapshot is then taken and the travel distance is converted into the momentum. This technique provides direct access to the complete momentum distribution $P(p ; t)$ and has been successfully applied in the recent experiments of Refs. [6-8].

In the presence of dynamical localization, the momentum distribution shows the exponential envelope of the atomic wavefunctions in momentum space, while a Gaussian envelope is expected when diffusive momentum spreading is induced by stationary timing or amplitude noise. In order to give an early indication of the effects of nonexponential decoherence, we compare in Fig. 3 the momentum distribution of Lévy kicked rotators with asymptotically stationary and nonstationary noise $(\alpha=2$ and $\alpha=1 / 2$, respectively). For $\alpha=2$, the strength of the noise is set to $\kappa=1 / 30000$, corresponding to a decoherence time $t_{c}=12.3 t^{*}$. For $t \lesssim t_{c}$ the distribution function is well described by a double-sided expo- 

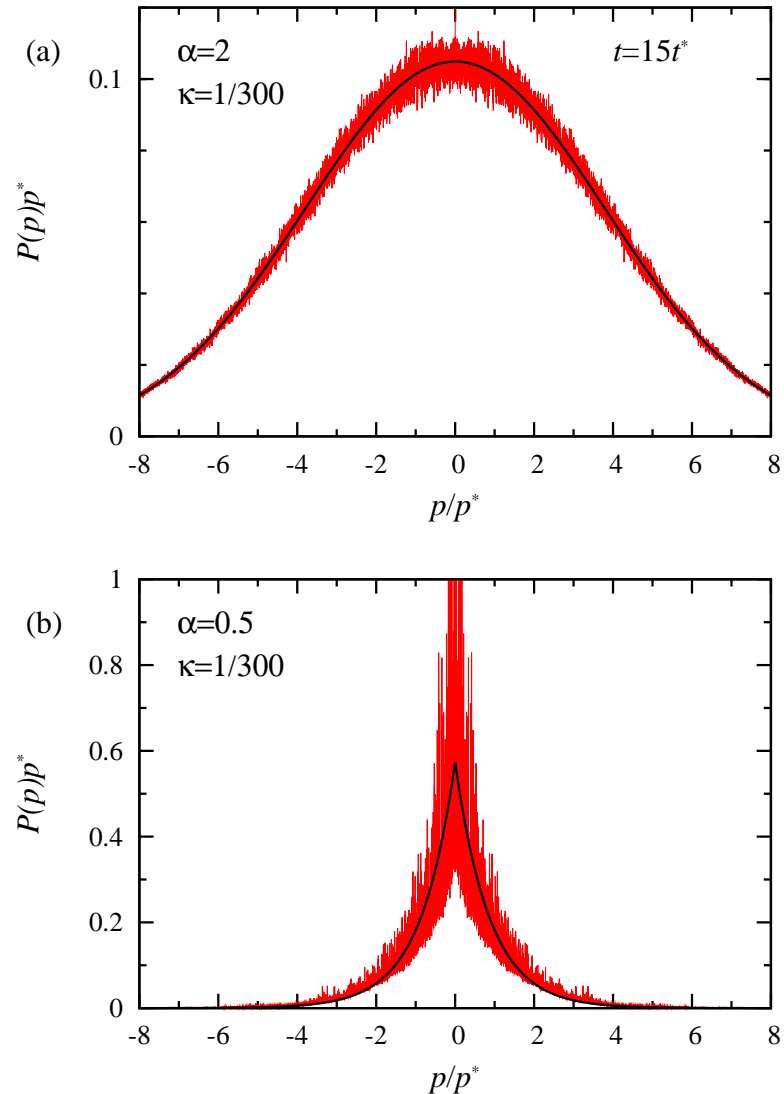

FIG. 4: (color online). Same as the left panels in Fig. 3, but for $\kappa=1 / 300\left(t_{c}=0.12 t^{*}\right)$, so that $t=15 t^{*} \gg t_{c}$.

nential function; when the time is increased it eventually evolves into a Gaussian distribution. This follows the expectations for conventional stationary noise. By contrast, for $\alpha=1 / 2$, where a well defined coherence time does not exist, the probability density keeps its doublesided exponential shape at all times. This is the case even though we increased the strength of the individual noise events by four orders of magnitude to $\kappa=1 / 3$, so that $t_{c}=0.00123 t^{*}$ is much reduced. This indicates that diffusive momentum spreading is never attained, even in the limit of very strong noise events.

Figure 4 shows the momentum distribution at $t=15 t^{*}$ for the more moderate noise strength $\kappa=1 / 300\left(t_{c}=\right.$ $\left.0.12 t^{*}\right)$ which is used in several subsequent figures in this work. For $\alpha=2.0$, the momentum distribution is always well-approximated by a Gaussian, while for $\alpha=0.5$ and $t>t^{*}$, it is well-approximated by a double-sided exponential function as soon as $t>t^{*}$.

We now proceed to characterize the evolution of the momentum distribution by two quantities, the variance var $p$ and the inverse participation ratio (IPR).

\section{A. Variance of momentum}

The temporal evolution of the momentum distribution $P(p ; t)$ has two origins. The first component is the spreading of the momentum wavefunction of an individual atom with respect to the mean momentum of the atom. This momentum spreading is characterized by the variance,

$$
\operatorname{var} p(t)=\overline{\left\langle p^{2}\right\rangle-\langle p\rangle^{2}}
$$

As indicated, this variance is computed from the quantum-mechanical expectation values, and then averaged over the noise. The second component of the evolution of the momentum distribution is the spreading of the mean momenta of the different atoms. Hence, in general, the variance (44) is smaller than the total variance computed from the momentum distribution $P(p ; t)$. In the particular case of the kicked rotator, however, this difference becomes negligible, due to the symmetry $(\theta, p) \rightarrow(-\theta,-p)$ which holds even in presence of the noise.

In the following, we first establish a general relation between the variance of the momentum of the noise-free and noisy systems, and then evaluate this relation for the Lévy kicked rotator. We will then find that nonexponential decoherence manifests itself in a subdiffusive spreading of the momentum.

As in the earlier theories of delocalization due to decoherence [15], the starting point of our considerations is the force-force correlation function,

$$
C\left(t^{\prime}, t^{\prime \prime}\right)=\overline{\left\langle K_{t^{\prime}} K_{t^{\prime \prime}} \sin \theta_{t^{\prime}} \sin \theta_{t^{\prime \prime}}\right\rangle}
$$

which is composed of the momentum increments in an individual time step, as given by Eq. (2). In the above equation, $\theta_{t}$ is the angle operator in the Heisenberg picture. The average includes an average over the initial momentum of the particle, so that the expectation value is replaced by the normalized trace $N^{-1} \operatorname{tr}_{N}$, where $N$ is the Hilbert space dimension.

The total change of the momentum is obtained by integrating the force over time. The variance therefore reads

$$
\operatorname{var} p(t)=\sum_{t^{\prime}, t^{\prime \prime}=0}^{t-1} C\left(t^{\prime}, t^{\prime \prime}\right)
$$

In order to establish the relation to the decoherence factor, the force-force correlation function is expanded in the basis of quasienergy eigenstates [15], 


$$
C\left(t^{\prime}, t^{\prime \prime}\right)=\overline{\sum_{\left\{r_{n}, s_{n}\right\}}^{\prime}\left\langle r_{t^{\prime}}|\sin \theta| s_{t^{\prime}}\right\rangle\left\langle s_{t^{\prime \prime}}|\sin \theta| r_{t^{\prime \prime}}\right\rangle K_{t^{\prime}} K_{t^{\prime \prime}} \prod_{l, m=t^{\prime \prime}}^{t^{\prime}-1}\left\langle r_{l}\left|F\left(K_{l}\right)\right| r_{l+1}\right\rangle *\left\langle s_{m+1}\left|F\left(K_{m}\right)\right| s_{m}\right\rangle .}
$$

The prime in the sum over quasienergy indices excludes the initial index $r_{t^{\prime \prime}}$, which originates from the normalized trace and hence instead is averaged over.

We first apply the random-phase approximation to the matrix elements of the sine kicking potential, which in principle selects three types of terms: (i) terms with $r_{t^{\prime}}=$ $r_{t^{\prime \prime}}, s_{t^{\prime}}=s_{t^{\prime \prime}}$, but $r_{t^{\prime}} \neq s_{t^{\prime}}$; (ii) terms with $r_{t^{\prime}}=s_{t^{\prime}}$, $r_{t^{\prime \prime}}=s_{t^{\prime \prime}}$, but $r_{t^{\prime}} \neq r_{t^{\prime \prime}}$; (iii) terms with $r_{t^{\prime}}=r_{t^{\prime \prime}}=s_{t^{\prime}}=$ $s_{t^{\prime \prime}}$.

The terms of type (ii) and (iii) involve the expectation value of the force, which vanishes in the specific case of the kicked rotator, thanks to the symmetry $(\theta, p) \rightarrow(-\theta,-p)$ (a careful analysis of such terms will be required for the purity, see Section V). For the terms of type (i), on the other hand, the product of transition matrix elements $\left\langle r_{l}\left|F\left(K_{l}\right)\right| r_{l+1}\right\rangle$ in quasienergy space exactly arranges itself into the definition of the decoherence factor (33). This correspondence also holds when $C\left(t^{\prime}, t^{\prime \prime}\right)$ is evaluated for $t^{\prime}<t^{\prime \prime}$, provided we define $\mathcal{D}\left(t^{\prime}, t^{\prime \prime}\right)=\mathcal{D}\left(t^{\prime \prime}, t^{\prime}\right)$.

For $t^{\prime}=t^{\prime \prime}$, the average over the noise also gives rise to a contribution to the typical force acting on the particle of the form $\overline{K_{t^{\prime}}^{2}}\left\langle\sin \theta^{2}\right\rangle=K^{2} / 2+(\kappa / 2) f\left(t^{\prime}\right)$, where $f(t)$ is the sprinkling distribution. Collecting all terms, we arrive at

$$
C\left(t^{\prime}, t^{\prime \prime}\right)=C_{0}\left(t^{\prime}, t^{\prime \prime}\right) \mathcal{D}\left(t^{\prime}, t^{\prime \prime}\right)+\frac{\kappa}{2} f\left(t^{\prime}\right) \delta_{t^{\prime}, t^{\prime \prime}},
$$

where $C_{0}\left(t^{\prime}, t^{\prime \prime}\right)$ is the force-force correlation function in the absence of noise. Besides the correction from the sprinkling distribution, which is small for weak noise, this recovers the result from the perturbative short-time theory in Ref. [15]. It follows that the correct extrapolation of the decoherence factor beyond the initial decay is given by Eq. (38).

The variance of the momentum follows when Eq. (48) is inserted into Eq. (46). It is then convenient to pass to a continuous time argument and perform integrations by parts, so that all force correlations are expressed in terms of the variance of the momentum in absence of the noise, which we denote by var $p_{0}(t)$. We then obtain as one of our main results the following expression,

$$
\begin{aligned}
& \operatorname{var} p(t)=\operatorname{var} p_{0}(t) \mathcal{D}(t, 0)+\frac{\kappa}{2} \overline{\mathcal{N}(t, 0)} \\
& +\int_{0}^{t} d s \operatorname{var} p_{0}(t-s) \partial_{s} \mathcal{D}(t, s) \\
& -\int_{0}^{t} d s \operatorname{var} p_{0}(s) \partial_{s} \mathcal{D}(s, 0) \\
& -\int_{0}^{t} d s^{\prime} \int_{0}^{s^{\prime}} d s^{\prime \prime} \operatorname{var} p_{0}\left(s^{\prime}-s^{\prime \prime}\right) \partial_{s^{\prime}} \partial_{s^{\prime \prime}} \mathcal{D}\left(s^{\prime}, s^{\prime \prime}\right)
\end{aligned}
$$

The second term on the right hand side can be neglected for small noise strength $\kappa$. Equation (49) expresses the momentum variance in presence of noise exclusively in terms of the variance in absence of the noise, and derivatives of the decoherence factor $\mathcal{D}\left(t^{\prime}, t^{\prime \prime}\right)$, which play the role of memory kernels. Because of the monotonicity properties of $\mathcal{D}\left(t^{\prime}, t^{\prime \prime}\right)$ all terms are positive. Since the result is expressed directly in terms of var $p_{0}$, Eq. (49) moreover accounts for all dynamical correlations of the noiseless dynamics. In particular, the random-phase approximation used in the derivation only relies on the complex quantum-dynamics of the noiseless system, but does not require that it displays dynamical localization.

In exploring the consequences of Eq. (49) for the kicked rotator, we combine Eq. (5) for the momentum variance in absence of the noise with the various

decoherence factors found in Section III. First, let us again make contact with the known results for stationary noise, for which the decoherence factor decays exponentially as given in Eq. (39). The corresponding momentum spreading is

$\operatorname{var} p=\frac{D^{*}}{1+t_{c} / t^{*}} t+\frac{D^{*} t^{*}}{\left(1+t^{*} / t_{c}\right)^{2}}\left[1-\exp \left(-t / t^{*}-t / t_{c}\right)\right]$.

This result was first proposed in Ref. [15] as one of two possible extrapolations of the perturbative short-time results, and has been successfully applied to quantify the decoherence process in the experiment of Ref. [7]. The present derivation shows that the other proposed extrapolation indeed was rightfully discarded. For large times, the second term approaches a constant and can be neglected in comparison to the first term, which increases linearly in time. Hence, for stationary noise when quantum coherence is lost exponentially, the momentum diffuses asymptotically with a renormalized diffusion constant $D^{*} /\left(1+t_{c} / t^{*}\right)$.

In the presence of asymptotically stationary noise generated by a renewal process with $\alpha>1$, the decoherence function is given by Eq. (40), in which the coherence time is modified by the mean waiting $\bar{\tau}$. This does not induce any qualitative change in the momentum spreading, which is still diffusive according to a renormalized diffusion constant $D^{*} /\left(1+\bar{\tau} t_{c} / t^{*}\right)$.

As $\alpha$ approaches unity from above, the decoherence time increases, and the quantum diffusion constant vanishes at $\alpha=1$. It is clear that this has to be accompanied by a qualitative change of the momentum spreading itself. For $\alpha<1$, the asymptotic spreading is dominated by the last term in Eq. (49), which can be approximated 
as

$$
\begin{aligned}
\operatorname{var} p(t) & \sim-\operatorname{var} p_{0}(\infty) \int_{0}^{t} d s^{\prime} \int_{0}^{s^{\prime}} d s^{\prime \prime} \partial_{s^{\prime}} \partial_{s^{\prime \prime}} \mathcal{D}\left(s^{\prime}, s^{\prime \prime}\right) \\
& =\frac{D^{*} t^{*}}{t_{c}} \overline{\mathcal{N}(t, 0)}
\end{aligned}
$$

Using the power-law asymptotics (13) of the inverse random time for $\alpha \leq 1$, we immediately find that the momentum spreads subdiffusively,

$$
\operatorname{var} p(t) \sim \frac{D^{*} t^{*}}{t_{c}} \frac{\sin \pi \alpha}{\pi c} t^{\alpha} .
$$

The full time dependence of the momentum variance of Lévy kicked rotators with asymptotically stationary or non-stationary noise is shown in Fig. 5. The upper panel shows the results for $\alpha=2$, for which the momentum spreading is diffusive. The classical diffusion is attained for moderate noise strength $\kappa \gtrsim 1 / 300$. The lower panel shows the results for $\alpha=1 / 2$, and confirms that in this case the spreading is subdiffusive, $\operatorname{var} p \propto t^{1 / 2}$. Note that this behavior persists even when the strength of the individual noise events is large. The theoretical predictions in the plots follow from Eq. (49), where $\operatorname{var} p_{0}(t)$ is taken from Eq. (5), while $\mathcal{D}\left(t^{\prime}, t^{\prime \prime}\right)$ is determined for Eq. (38) [i.e., the moment-generating function of $\mathcal{N}\left(t^{\prime}, t^{\prime \prime}\right)$ for the Yule-Simon distribution is evaluated at $\left.z=-1 / t_{c}=-\kappa / 2 \hbar^{2}\right]$. There is good agreement between the numerical results and the theoretical predictions.

\section{B. Inverse participation ratio}

In this section we study an alternative measure of the momentum spreading, the inverse participation ratio (IPR), and show that this quantity also reveals the existence of two distinct (exponential and non-exponential) decoherence regimes. The inverse participation ratio is defined as

$$
\operatorname{IPR}(t)=\sum_{n} \overline{\left|\psi_{n}(t)\right|^{4}}
$$

where $\psi_{n}$ are the amplitudes of the wavefunction in a fixed basis. The IPR can be interpreted as a measure for the inverse dimension of an effective Hilbert space explored by the quantum dynamics. In general, the IPR is basis dependent. In the following, we provide a quasiclassical estimate of the IPR by introducing basis states which cover Planck cells of area $2 \pi \hbar$ in phase-space and are centered at phase-space positions $\left(\theta_{n}, p_{n}\right)$. This estimate turns out to be independent on the details of the quasiclassical basis.

For a given time, the complex amplitudes $\psi_{n}$ fluctuate as $n$ is varied, corresponding to speckle noise in the phasespace position $\left(\theta_{n}, p_{n}\right)$. In order to quantify these fluctuations, we introduce a phase-space function $P\left(\theta_{n}, p_{n} ; t\right)$

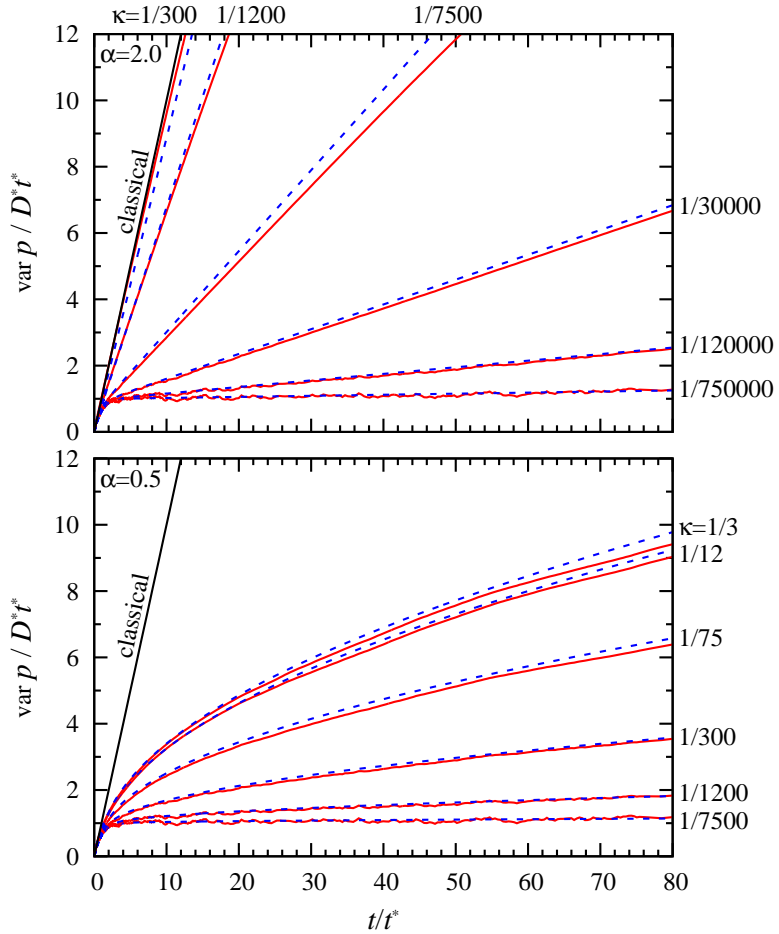

FIG. 5: (color online). Time dependence of the momentum spreading var $p(t)$ for kicked rotators which are subjected to Lévy noise generated by a Yule-Simon distribution with noise exponent $\alpha=2.0$ (panel a) and $\alpha=0.5$ (panel b). The results of numerical computations (solid curves) are compared to the theoretical prediction from Eq. (49) (dashed curves). The straight solid line labelled "classical" is the classical diffusion.

such that the statistical average of the squared amplitude is

$$
\overline{\left|\psi_{n}(t)\right|^{2}} \equiv 2 \pi \hbar P\left(\theta_{n}, p_{n} ; t\right)
$$

By assuming that the real and imaginary parts of $\psi_{n}$ are Gaussian distributed and independent, we have

$$
\overline{\left|\psi_{n}(t)\right|^{4}}=2{\overline{\left|\psi_{n}(t)\right|^{2}}}^{2} .
$$

In the quasiclassical approximation, the function $P(\theta, p ; t)$ is now identified with a smooth, properly normalized phase space density with continuous variables $\theta$ and $p$. By combining Eqs. (54) and (55), the IPR can then be written in the form

$$
\operatorname{IPR}(t)=\int \frac{d \theta d p}{2 \pi \hbar} \times 2[2 \pi \hbar P(\theta, p ; t)]^{2} .
$$

The above expression is invariant under canonical transformations of the phase-space variables and hence exhibits the advertised independence of details of the quasiclassical basis.

In the Lévy kicked rotator, the quasiclassical probability density becomes $\theta$-independent after a short time, so that to a good approximation $P(\theta, p ; t) \simeq P(p ; t) / 2 \pi$. 
This leads to a compact expression for the IPR,

$$
\operatorname{IPR}(t)=2 \hbar \int d p P^{2}(p ; t)
$$

The integral in Eq. (57) can be calculated in two simple cases: For a Gaussian profile, $P(p ; t)=$ $(2 \pi \operatorname{var} p(t))^{-1 / 2} \exp \left[-p^{2} / 2 \operatorname{var} p(t)\right]$, typical for diffusive spreading, we obtain

$$
\operatorname{IPR}(t)=\frac{\hbar}{\sqrt{\pi \operatorname{var} p(t)}} \quad(\text { Gaussian profile })
$$

On the other hand, for a double-sided exponential probability distribution, $P(p ; t)=(\lambda / 2) \exp (-\lambda|p|)$ with $\lambda=$ $\sqrt{2 / \operatorname{var} p}$, we find

$$
\operatorname{IPR}(t)=\frac{\hbar}{\sqrt{2 \operatorname{var} p(t)}} \quad(\text { exponential profile }) .
$$

Interestingly, in both Eqs. (58) and (59) the IPR is proportional to $\hbar / \sqrt{\operatorname{var} p(t)}$. In view of Eq. (52), we hence expect that the asymptotic decay of the IPR is a powerlaw with exponent $-1 / 2$ for $\alpha>1$, while for $\alpha<1$ the exponent is reduced to $-\alpha / 2$. These predictions are confirmed by the numerical results shown in Fig. 6 . We can therefore conclude that the quasiclassical expressions (58) and (59) offer a good quantitative estimate of the IPR of the Lévy kicked rotator.

\section{PURITY AND FIDELITY}

A convenient quantity to further elucidate the loss of coherence in a quantum-dynamical system is the fidelity $[22,23,34,35]$,

$$
\mathcal{F}(t)=\left|\left\langle\psi(t) \mid \psi^{\prime}(t)\right\rangle\right|^{2},
$$

where the states $\psi(t)$ and $\psi^{\prime}(t)$ start out from the same initial condition $\psi(0)=\psi^{\prime}(0)$, but are propagated with different realizations of the noise. An extensive review of the intricate features of the fidelity can be found in Ref. [24].

A remarkable property of the fidelity is that it can be directly probed in an echo experiment [25]. In the latter, the quantum state is first let to evolve up to a certain time $t$ at which the dynamics is reversed, e.g., by applying a pulse that inverts the momentum $(p \rightarrow-p)$. When at time $2 t$ the momentum-reverting pulse is applied again, the system approximately returns into its initial state. The fidelity characterizes the quality of this echo, which is never perfect because of imperfections in the momentum-reverting pulse and the noise in the dynamics. The destruction of phase coherence in a quantum system such as the kicked rotator can therefore be directly studied in echo-like experiments. To our knowledge such a measurement has not yet been performed in kicked rotator experiments. However, recently several
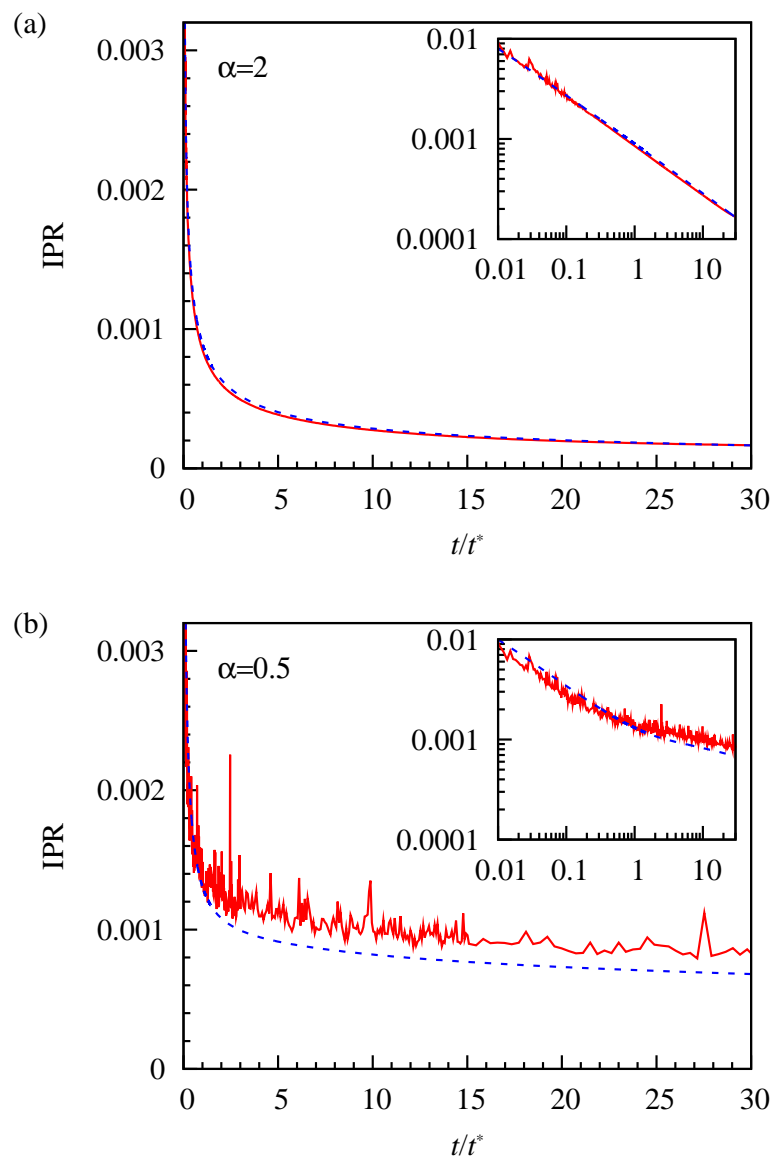

FIG. 6: (color online). Time dependence of the inverse participation ratio IPR for kicked rotators which are subjected to Lévy noise generated by a Yule-Simon distribution with $\kappa=1 / 300$. The main panels are single-logarithmic while the insets show the same data in a double-logarithmic presentation. (a) Noise with exponent $\alpha=2.0$, corresponding to a decoherence time $t_{c}=0.12 t^{*}$ (where $t^{*}$ is the localization time) so that the momentum distribution $P(p ; t)$ is always well-approximated by a Gaussian. The dashed line is the quasi-classical prediction (58). (b) Noise with exponent $\alpha=0.5$, where for $t>t^{*}$ the momentum distribution $P(p ; t)$ is always well-approximated by a double-sided exponential function. The dashed line is the quasi-classical prediction (59).

proposals on how to measure the fidelity in atom-optical experiments have been put forward [26-28].

In the present section, we study various statistical aspects of the fidelity for the case that its decay is induced by Lévy noise.

\section{A. Purity}

The statistical average of the fidelity

$$
\overline{\mathcal{F}(t)}=\operatorname{tr} \rho^{2}(t)
$$

is formally equivalent to the so-called purity of the statistical mixture of the perturbed states, which is described 
by the density operator

$$
\rho(t)=\overline{|\psi(t)\rangle\langle\psi(t)|} .
$$

The average is over the amplitude and timing noise generated by the renewal process. The initial state is fixed, so that the purity starts at $\operatorname{tr} \rho^{2}(0)=1$. For completely incoherent superpositions the purity tends to the value $1 / N$, where $N$ is the Hilbert space dimension.
In the following, we show that the initial and intermediate decay of the purity is directly related to the decoherence function $\mathcal{D}(t)$. For the long-time asymptotics, we moreover establish a connection to the inverse participation ratio.

In the quasienergy basis, starting from Eq. (61), the purity can be expanded as

$\operatorname{tr} \rho^{2}(t)=\overline{\sum_{\left\{r_{n}, s_{n}, r_{n}^{\prime}, s_{n}^{\prime}\right\}}^{\prime} \psi_{r_{0}} \psi_{r_{0}^{\prime}}^{*} \psi_{s_{0}}^{*} \psi_{s_{0}^{\prime}} \prod_{l, m, l^{\prime}, m^{\prime}=0}^{t-1}\left\langle r_{l+1}\left|F\left(K_{l}\right)\right| r_{l}\right\rangle\left\langle r_{l^{\prime}+1}^{\prime}\left|F\left(K_{l^{\prime}}^{\prime}\right)\right| r_{l^{\prime}}^{\prime}\right\rangle *\left\langle s_{m+1}\left|F\left(K_{m}\right)\right| s_{m}\right\rangle^{*}\left\langle s_{m^{\prime}+1}^{\prime}\left|F\left(K_{m^{\prime}}^{\prime}\right)\right| s_{m^{\prime}}^{\prime}\right\rangle}$,

where the prime in the sum denotes the constraint $r_{t}=$ $r_{t}^{\prime}, s_{t}=s_{t}^{\prime}$. Furthermore, $\psi_{r}$ is the initial wavefunction, expanded in the quasienergy eigenbasis, and the values $K_{l}$ and $K_{l}^{\prime}$ are obtained from independent realizations of the renewal process, which differ both in the timing as well as in the detunings of the noisy kicks.

We first apply the random-phase approximation to the expansion coefficients $\psi_{r}$, which selects three groups of terms: (i) terms with $r_{0}=r_{0}^{\prime}, s_{0}=s_{0}^{\prime}$, but $r_{0} \neq s_{0}$; (ii) terms with $r_{0}=s_{0}, r_{0}^{\prime}=s_{0}^{\prime}$, but $r_{0} \neq r_{0}^{\prime}$; (iii) terms with $r_{0}=r_{0}^{\prime}=s_{0}=s_{0}^{\prime}$.

For each class of terms, the random-phase averages over the detunings and timing of the noisy kicks can be carried out following the procedure which we applied to the decoherence factor and the survival probability in quasienergy space, described in Section III. For the terms of group (i), this procedure selects only diagonal matrix elements with fixed $r_{n}=r_{n}^{\prime} \equiv r, s_{n}=s_{n}^{\prime} \equiv s$, just as encountered for the decoherence factor. Since we now deal with two independent renewal processes, they deliver a factor $\exp \left[-\mathcal{N}(t, 0) / t_{c}+\mathcal{N}^{\prime}(t, 0) / t_{c}\right]$ which depends on the sum of inverse random times in the propagation of the two states. After averaging over the timing noise of the two independent renewal processes, these terms therefore deliver a factor $\mathcal{D}^{2}(t, 0)$.

Among the terms of group (ii), the random-phase approximation requires to pair all amplitudes to probabilities, $r_{n}=s_{n}, r_{n}^{\prime}=s_{n}^{\prime}$. In the calculation of the variance var $p(t)$, these terms could be neglected because they were multiplied by the vanishing expectation value of the detuned force. We shall denote these contributions by $\mathcal{S}_{r \rightarrow s, \mathrm{cl}}(t, 0)$.

Among the terms of group (iii), both terms which are completely diagonal (type i) as well as terms which are paired to probabilities (type ii) survive. A neat separation of these terms follows when we require that for $r=s$, the terms are called of type (ii) when they contain at least one quasienergy change. Appropriately attribut- ing these terms to the groups (i) and (ii), the purity then takes the form

$$
\operatorname{tr} \rho^{2}(t)=\sum_{r, s}\left|\psi_{r}\right|^{2}\left|\psi_{s}\right|^{2}\left[\mathcal{D}^{2}(t, 0)+\mathcal{S}_{r \rightarrow s, \mathrm{cl}}(t, 0)\right],
$$

where the restriction $r \neq s$ is now lifted.

For the term proportional to $\mathcal{D}^{2}(t, 0)$ we can now exploit the normalization of the initial wavefunction. Looking at the second term, it easy to see that these contributions can be neglected for short times $t \ll t_{c}$, since per definition they contain at least one quasi-energy transition. The significance of these terms becomes apparent when one re-evaluates the purity for long times. For this we start from the general expression,

$$
\operatorname{tr} \rho^{2}(t)=\sum_{n m} \overline{\psi_{n}^{*}(t) \psi_{n}^{\prime}(t) \psi_{m}^{\prime *}(t) \psi_{m}(t)}
$$

and adopt the quasi-classical basis in which we computed the IPR (see Section IV B). For large times $t \gg t_{c}$ it is reasonable to assume that the relative phases of the wavefunction expansion coefficients $\psi_{n}$ and $\psi_{n}^{\prime}$ in this basis are completely randomized. Assuming furthermore $\left|\psi_{n}\right|^{2} \approx\left|\psi_{n}^{\prime}\right|^{2}$, we find that $\operatorname{tr} \rho^{2}(t)$ asymptotically approaches the IPR as defined in Eq. (53). The terms of group (ii) are therefore associated to the eventual saturation of the purity at the inverse of the effective Hilbert space dimension. We hence arrive at our main result for the purity,

$$
\operatorname{tr} \rho^{2}(t) \approx \mathcal{D}^{2}(t, 0)+\operatorname{IPR}(t) .
$$

The initial and intermediate decay of the purity is given by the first term, which is simply the square of the decoherence function $\mathcal{D}(t, 0)$. For stationary noise the initial decay is thus exponential, $\operatorname{tr} \rho^{2}(t)=\exp \left(-2 t / t_{c}\right)$. A similar behavior is predicted for Lévy noise with $\alpha>1$, for which $t_{c}$ replaced by $\bar{\tau} t_{c}$. For nonstationary noise with $\alpha<1$, Eq. (41) predicts that the initial and intermediate decay shows the functional features of the 


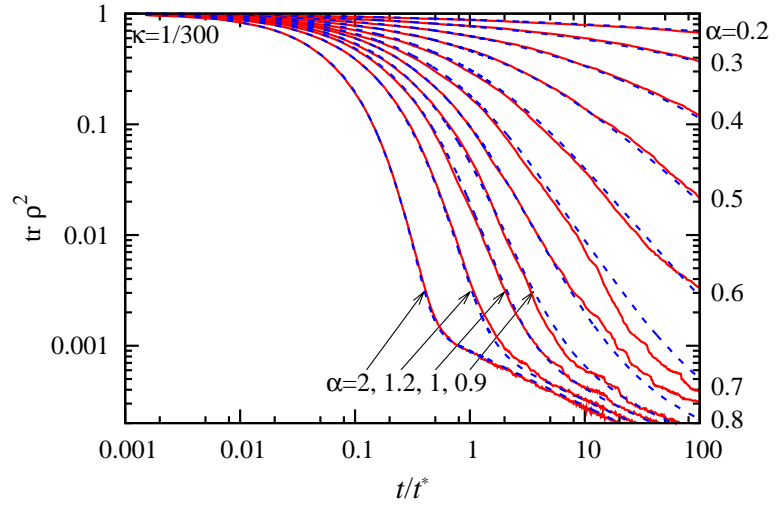

FIG. 7: (color online). Time dependence of the purity $\operatorname{tr} \rho^{2}$ of Lévy kicked rotators for different noise exponents $\alpha$ and fixed noise strength $\kappa=1 / 300$. The results of numerical simulations (solid curves) are compared to the theoretical predictions from Eq. (66) (dashed curves)

squared Mittag-Leffler function, i.e., a stretched exponential which for later times crosses over to a power law with exponent $-2 \alpha$.

The long time behavior of the purity is given by the saturation at the inverse effective Hilbert space dimension, which we estimate in terms of the inverse participation ratio. According to Eqs. (58) and (59), this term is proportional to $[\operatorname{var} p(t)]^{-1 / 2}$. For $\alpha>1$ the exponential decay therefore asymptotically crosses over to a powerlaw decay with exponent $-1 / 2$. As discussed before, for $\alpha<1, \mathcal{D}^{2}(t)$ itself already crosses over from a stretched exponential to a power law with exponent $-2 \alpha$. As a consequence of the subdiffusive momentum spreading, this intermediate power law is asymptotically replaced by a slower power law with exponent $-\alpha / 2$.

As a function of the inverse random time $\mathcal{N}(0, t)$, the crossover to the asymptotic power-law of the saturation background sets in at $\mathcal{N}(0, t) \approx t_{c} \ln t^{*}$. For $\alpha>1$, this condition translates into a well-defined crossover time $t \approx$ $\bar{\tau} t_{c} \ln t^{*}$. For $\alpha<1$, however, the nonergodicity of Lévy noise entails that the crossover is blurred.

The numerical results presented in Fig. 7 confirm the quantitative and qualitative predictions of Eq. (66) for the purity of the Lévy kicked rotator. For $\alpha>1$ the initial decay is exponential and the asymptotic decay is algebraic with exponent $-1 / 2$. The background contribution is here clearly visible and displays the predicted power-law decay. For $\alpha<1$ the decay is a stretched exponential which crosses over to an algebraic decay with exponent $-2 \alpha$. Because of this significant slow-down of the decoherence, the eventual crossover of the exponent to $-\alpha / 2$ is only observed in the numerical data for $\alpha \gtrsim 0.7$; for smaller values of $\alpha$, the background contribution is hardly discernible.

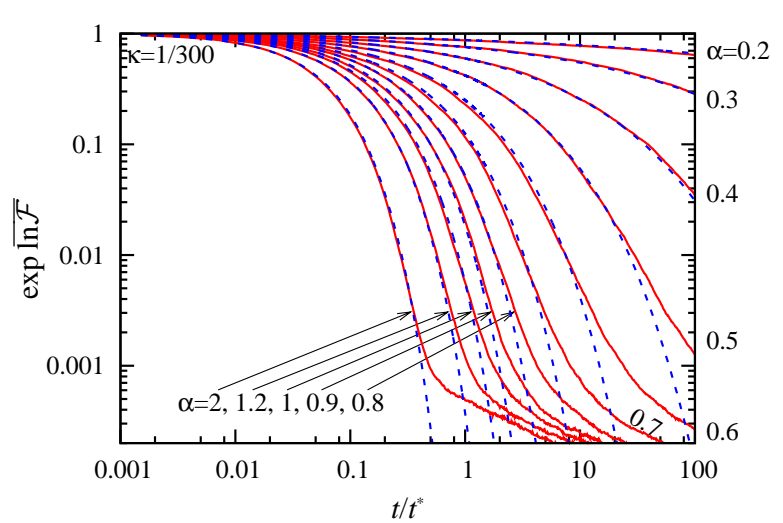

FIG. 8: (color online). Averaged logarithm of the fidelity of Lévy kicked rotators for different noise exponents $\alpha$ and fixed noise strength $\kappa=1 / 300$. The results of numerical simulations (solid curves) are compared to the theoretical predictions from Eq. (68) (dashed curves), which does not account for the saturation background.

\section{B. Averaged logarithm of the fidelity}

For a system with complex quantum dynamics such as the kicked rotator, it is often observed that the fidelity fluctuates significantly from realization to realization [34], and from Fig. 7 it is clear that this tendency is exacerbated for nonergodic noise. These fluctuations are suppressed when one instead considers the averaged logarithm $\overline{\ln \mathcal{F}(t)}$ of the fidelity.

By passing from the fidelity to its logarithm, the multiplicative suppression of coherence per noise event is converted into additive contributions. For initial and intermediate times, the averaged logarithm hence probes the mean number of noise events in both noise realizations,

$$
\overline{\mathcal{N}(0, t)}+\overline{\mathcal{N}^{\prime}(0, t)}=2 \int_{0}^{t} f\left(t^{\prime}\right) d t^{\prime}
$$

where $f(t)$ is the sprinkling distribution. This leads to the prediction

$\overline{\ln \mathcal{F}(t)}=-\frac{2}{t_{c}} \int_{0}^{t} f\left(t^{\prime}\right) d t^{\prime} \quad$ (short and intermediate times),

while asymptotically a saturation background should set in (we do not give an estimate of this background).

The validity of this prediction is confirmed by the numerical results in Fig. 8, which shows the averaged logarithm in a representation that makes it readily comparable to the results for the purity (Fig. 7). There is good agreement for small and intermediate time, until saturation sets in, which is not incorporated in Eq. (68). Two noteworthy observations in the comparison to the purity are first the inequality $\exp (\overline{\ln \mathcal{F}})<\operatorname{tr} \rho^{2}$, and secondly the fact that the statistical fluctuations are now distinctively suppressed. This indicates that the logarithm of the fidelity probes typical noise realizations, while the 
purity is influenced by rare noise realizations in which coherence is exceptionally well preserved.

\section{DISCUSSION AND CONCLUSIONS}

In this concluding section, we would like to situate our work in the general context of the study of anomalous diffusion and nonexponential relaxation. The investigation of diffusion in complex environments enjoys a long history starting with the seminal work of Scher and Montroll on dispersive transport in amorphous semiconductors [36]. Subsequently, extensive work on non-Brownian motion and nonexponential decay induced by Lévy noise in space and/or in time in classical systems has been carried out, most prominently using the powerful Continuous Time Random Walk (CTRW) formalism (for a review see Ref. [37]). In the last few years, this line of research has been extended to quantum systems. The novel aspect of these developments is the possibility to study the interplay of complex dynamics and quantum phenomena. Thus the interaction with a composite environment with extra degrees of freedom, and the entanglement of the quantum system with the latter, has for instance be shown to lead to power-law decay [38]. The relaxation of a quantum two-level system subjected to stationary and nonstationary power-law noise has been respectively examined in Refs. [39] and [40]. In the latter work, the presence of aging dephasing has been demonstrated. Additionally, anomalous fast decoherence induced by spatial Lévy noise, stemming from a chaotic random-matrix environment, has been investigated in Ref. [41].

Our approach in the present work has been to use the quantum kicked rotator as a powerful tool to explore decoherence and its interplay with complex quantum dynamics, in particular, nonstationarity. The latter property is well-known from the physics of disordered or glassy materials [42]. Taking advantage of the unique degree of tunability of the atom-optical realization of the kicked rotator, we have put forward a way to engineer a complex reservoir, providing full control over the stationarity of the environment or the absence thereof. Specifically, we have proposed to simulate the coupling to a complex reservoir with the help of Lévy noise with variable exponent. In this manner, we extend usual quantum reservoir engineering in the spirit of the early studies of anomalous diffusion in complex media based on the random walk concept. The renewal process that we investigate can actually be regarded as corresponding to a CTRW with power-law waiting time, but with a renormalized diffusion range given by the localization length of the kicked rotator.

At this point, it is appropriate to mention that anomalous diffusion has been shown before to occur in quantum kicked rotators subjected to deterministic aperiodic kicking. More precisely, subballistic wave packet spreading induced by quasiperiodic Fibonacci kick sequences has been established both away and at resonance $[43,44]$.
The effect of Lévy noise at resonance has also been the subject of a recent study [45]. An important difference to the present study is the fact that these studies do not consider the simultaneous presence of the periodic kicks in the intervals between the noise events. As a consequence, the classical momentum dynamics for the quasiperiodically kicked systems is subdiffusive, and hence does not differ qualitatively from the quantum dynamics. The protocol proposed in the present paper stipulates the presence of such periodic kicks, which induce diffusive classical momentum dynamics, while quantum mechanically they drive the system towards localization. The ensuing qualitative difference between the noiseless quantum and classical dynamics indeed provides the terrain in which we explore the consequences of decoherence.

The theory of decoherence that we have developed in this paper combines the properties of Lévy noise, encoded in the generating function of the number of noisy events (14), and a random-phase approximation, which exploits the complex quantum dynamics of the rotator appearing in the quasienergy transition amplitudes (30). It is worth emphasizing that it is nonperturbative (with respect to time) and applies both to stationary and nonstationary noise.

One of the central results of our paper, contained in Eq. (41), is the demonstration of a regime of nonexponential loss of phase coherence when the noise is nonstationary (Lévy exponent $\alpha<1$ ). This regime is characterized by an infinite coherence time, indicating that classical behavior can never be attained, even though the amplitude of the noise is dramatically increased by several orders of magnitude. This remarkable property finds its origin in the divergence of the mean waiting time between random kicks and hence in the absence of a characteristic time scale in the engineered reservoir.

The existence of a regime of slow decoherence has direct consequence for experimentally accessible physical quantities of the kicked rotator. We have first shown that the momentum spreading (52) is subdiffusive and that the momentum profile retains a double-sided exponential shape, see Fig. 3. These asymptotic features are in stark contrast to those in the usual decoherence regime, here realized for Lévy exponent $\alpha>1$, which is characterized by normal diffusion and a Gaussian profile. The momentum distribution is routinely measured in kicked rotator experiments. In the atom-optical experiments $[6,7]$, the variance of the momentum is accessible up to times of the order of ten break times $t^{*}$, indicating that the subdiffusive dynamics depicted in Fig. 5 is measurable with current setups.

Two other important quantities that we have considered are the fidelity and the purity (or average fidelity), which are more traditional quantities for the characterization of decoherence. Here again we have found clear signatures of the nonexponential decoherence regime. In Eq. (66) we have established a direct connection between the purity on the one hand and the decoherence factor and the inverse participation on the other. As a conse- 
quence, we could show that for $\alpha<1$ the decay of the purity evolves from a stretched exponential to an algebraic decay, while $\alpha>1$ the decay changes from exponential to algebraic with exponent $-1 / 2$ (see Fig. 8). The fidelity can be directly measured in echo experiments, see for example Ref. [40], and various measurement schemes have been proposed for kicked rotator experiments [26-28]. We also mention that we have found a direct relationship (68) between the averaged logarithm of the fidelity and the sprinkling distribution of the renewal process.
Our concluding message is that reservoir engineering can be extended to mimic the coupling to complex nonstationary environments, allowing to control in a precise manner the loss of phase coherence and reveal hitherto unexplored decoherence scenarios.

This work was supported by the European Commission, Marie Curie Excellence Grant MEXT-CT-2005023778 (Nanoelectrophotonics), the Emmy Noether program of the DFG, contract LU1382/1-1, and the cluster of excellence Nanosystems Initiative Munich (NIM).
[1] W. H. Zurek, Rev. Mod. Phys. 75, 715 (2003).

[2] E. Joos, H. D. Zeh, C. Kiefer, D. Giulini, J. Kupsch, I.-O. Stamatescu, Decoherence and the Appearence of a Classical World in Quantum Theory, 2nd edition (Springer, Berlin, 2003).

[3] J. F. Poyatos, J. I. Cirac, and P. Zoller, Phys. Rev. Lett. 77, 4728 (1996).

[4] M. Brune, E. Hagley, J. Dreyer, X. Maître, A. Maali, C. Wunderlich, J. M. Raimond, and S. Haroche, Phys. Rev. Lett. 77, 4887 (1996).

[5] C. J. Myatt, B. E. King, Q. A. Turchette, C. A. Sackett, D. Kielpinski, W. M. Itano, C. Monroe, D. J. Wineland, Nature (London) 403, 269 (2000).

[6] H. Ammann, R. Gray, I. Shvarchuck, and N. Christensen, Phys. Rev. Lett. 80, 4111 (1998).

[7] B. G. Klappauf, W. H. Oskay, D. A. Steck, and M. G. Raizen, Phys. Rev. Lett. 81, 1203 (1998).

[8] W. H. Oskay, D. Steck, and M. Raizen, Chaos, Solitons and Fractals 16, 409 (2003).

[9] B. V. Chirikov, Phys. Rep. 52, 263 (1978).

[10] R. Graham, M. Schlautmann, and P. Zoller, Phys. Rev. A 45, R19 (1992).

[11] F. L. Moore, J. C. Robinson, C. F. Bharucha, B. Sundaram, and M. G. Raizen, Phys. Rev. Lett. 75, 4598 (1995).

[12] S. Fishman, D. R. Grempel, and R. E. Prange, Phys. Rev. Lett. 49, 509 (1982).

[13] F. M. Izrailev, Phys. Rep. 196, 299 (1990).

[14] E. Ott, T. M. Antonsen, and J. D. Hanson, Phys. Rev. Lett. 53, 2187 (1984).

[15] D. Cohen, Phys. Rev. Lett. 67, 1945 (1991); Phys. Rev. A 44, 2292 (1991).

[16] The decoherence-induced quantum-to-classical crossover has to be distinguished from the semiclassical crossover induced by a small Planck's constant, $\hbar \rightarrow 0$. For the interplay of both crossovers see J. Tworzydło, A. Tajic, H. Schomerus, P. W. Brouwer, and C. W. J. Beenakker, Phys. Rev. Lett. 93, 186806 (2004); A. Altland, P. W. Brouwer, and C. Tian, Phys. Rev. Lett. 99, 036804 (2007); R. S. Whitney, P. Jacquod, and C. Petitjean, Phys. Rev. B 77, 045315 (2008).

[17] M. F. Shlesinger, G. M. Zaslavsky, and J. Klafter, Nature 363, 31 (1993).

[18] E. Lutz, Phys. Rev. Lett. 93, 190602 (2004).

[19] G. Margolin and E. Barkai, Phys. Rev. Lett. 94, 080601 (2005).

[20] A. Erdélyi, Higher Transcendental Functions, Vol. 3 (McGraw-Hill, New York, 1955).

[21] H. Schomerus and E. Lutz, Phys. Rev. Lett. 98, 260401
(2007).

[22] R. A. Jalabert and H. M. Pastawski, Phys. Rev. Lett. 86, 2490 (2001).

[23] F. M. Cucchietti, H. M. Pastawski, and R. A. Jalabert, Phys. Rev. B 70, 035311 (2004).

[24] T. Gorin, T. Prosen, T. H. Seligman, M. Znidaric, Phys. Rep. 435, 33 (2006).

[25] R. Schäfer, H.-J. Stöckmann, T. Gorin, and T. H. Seligman, Phys. Rev. Lett. 95, 184102 (2005).

[26] S. A. Gardiner, J. I. Cirac, and P. Zoller, Phys. Rev. Lett. 79, 4790 (1997); 80, 2968 (1998).

[27] F. Haug, M. Bienert, W. P. Schleich, T. H. Seligman, and M. G. Raizen, Phys. Rev. A 71, 043803 (2005).

[28] S. Wimberger and A. Buchleitner, J. Phys. B 39, L145 (2006).

[29] C. F. F. Karney, A. B. Rechester, and R. B. White, Physica D 4, 425 (1982); A. B. Rechester and R. B. White, Phys. Rev. Lett. 44, 1586 (1980); A. B. Rechester, M. N. Rosenbluth, and R. B. White, Phys. Rev. A 23, 2664 (1981).

[30] B. V. Chirikov, F. M. Izrailev and D. L. Shepelyansky, Sov. Sci. Rev. C 2, 209 (1981); D. L. Shepelyansky, Physica D 28, 103 (1987).

[31] W. Feller, An Introduction to Probability Theory and Its Applications (Wiley, New York, 1968).

[32] A. Piryatinska, A. I. Saichev, and W. A. Woyczynski, Physica A 349, 375 (2005).

[33] H. A. Simon, Biometrika 42, 425 (1955).

[34] P. G. Silvestrov, J. Tworzydło, and C. W. J. Beenakker, Phys. Rev. E 67, 025204(R) (2003).

[35] P. Jacquod, P. G. Silvestrov, and C. W. J. Beenakker, Phys. Rev. E 64, 055203(R) (2001).

[36] H. Scher and E. W. Montroll, Phys. Rev. B 12, 2455 (1975).

[37] R. Metzler and J. Klafter, Phys. Rep. 339, 1 (2000).

[38] A. A. Budini and H. Schomerus, J. Phys. A 38, 9251 (2005).

[39] I. Goychuk and P. Hänggi, Chem. Phys. 324, 160 (2006).

[40] J. Schriefl, M. Clusel, D. Carpentier, and P. Degiovanni, Europhys. Lett. 69, 156 (2005); Phys. Rev. B 72, 035328 (2005).

[41] E. Lutz, Phys. Lett. A 293, 123 (2002).

[42] R. Richert and A. Blumen, Disorder Effects on Relaxational Processes (Springer, Berlin, 1994).

[43] G. Casati, G. Mantica, and D. L. Shepelyansky, Phys. Rev. E 63, 066217 (2001).

[44] A. Romanelli, R. Siri, and V. Micenmacher, Phys. Rev. E 76, 037202 (2007)

[45] A. Romanelli, A. Auyuanet, R. Siri, and V. Micen- 
macher, Phys. Lett. A 365, 200 (2007). 